\title{
Peripheral nerve injury in rats induces alternations in choice behavior associated with food reinforcement
}

\author{
Ken-ichiro Hayashida ${ }^{1}\left[\right.$ ] James C. Eisenach ${ }^{2} \cdot$ Masahito Kawatani $^{1} \cdot$ Thomas J. Martin $^{2}$
}

Received: 26 February 2019 / Accepted: 25 June 2019 / Published online: 2 July 2019

(c) The Physiological Society of Japan and Springer Japan KK, part of Springer Nature 2019

\begin{abstract}
Operant methods that allow animals to avoid painful stimuli are interpreted to assess the aversive quality of pain; however, such measurements require investigator-initiated stimuli to animals. Here we developed a shuttle maze test to repeatedly assess activity associated nociception without forced stimulation. Rats ambulate back and forth between two treat feeders by taking either a short route with a prickly surfaced arch or a longer route with a smooth floor. L5-L6 spinal nerve ligation (SNL) reduced the preference for the short route with the arch, correlated with hypersensitivity in the hind paw. Oral gabapentin restored the short route preference and reduced hypersensitivity in SNL rats, and blockade of spinal $\alpha 2$-adrenoceptors reduced gabapentin's effects on hypersensitivity but not on preference index. These results suggest that SNL injury alters behavior in the shuttle maze test and that the shuttle maze test shows comparable results to reflexive hypersensitivity after SNL in magnitude and response to gabapentin.
\end{abstract}

Keywords Choice behavior $\cdot$ Food reward $\cdot$ Neuropathic pain $\cdot$ Rats $\cdot$ Gabapentin

\section{Introduction}

Neuropathic pain arising from traumatic, metabolic, or oncologic injury to peripheral nerves is often accompanied by hypersensitivity to mechanical stimuli. The traditional measurements of mechanical hypersensitivity in animals are simple reflexes or innate responses, such as withdrawing, shaking, or licking the stimulated paw. These evoked responses mainly rely on spinal reflexes and are useful to assess the sensory component of hypersensitivity; however, they are unlikely to assess the affective component of hypersensitivity [1] and cannot be used to infer a pain experience. In addition, stimulus delivery by the experimenter makes those measurements highly susceptible to experimenter bias [2].

The place escape avoidance paradigm (PEAP) has been useful in assessing the aversive quality of mechanical stimuli in rodents with unilateral neuropathic and inflammatory

Ken-ichiro Hayashida

hayashida@med.akita-u.ac.jp

1 Department of Neurophysiology, Akita University School of Medicine, Akita 010-8543, Japan

2 Pain Mechanisms Laboratory, Department of Anesthesiology, Wake Forest School of Medicine, Winston-Salem, NC 27157, USA hypersensitivity [3, 4]. In the original PEAP [3], the animal is allowed to move between a light and a dark area, and receives repeated mechanical stimulations in the hypersensitive paw when in the dark area and the non-hypersensitive paw when in the light area, so that the animal shows a preference for either area based on a conflict of aversion between light and pain. The modified version of PEAP developed by Pratt et al. consists of two white areas with or without vertical black lines to which normal animals show no place preference, but animals with paw inflammation prefer the area without noxious mechanical stimuli as seen in the original PEAP [4]. Although the PEAP has been an important methodological contribution to pain research, it involves stimulation delivery by the experimenter and can induce fear, anxiety, or stress in animals, all of which can affect nociceptive threshold or mask pain behaviors $[5,6]$.

Here we developed a new operant method, the shuttle maze test, to assess the aversive quality of mechanical stimuli in rats after L5-L6 spinal nerve ligation (SNL) without externally applied stimuli by the experimenter. The shuttle maze test is based on the motivation for chocolate flavor cereal as a treat, and animals learn to associate alternate availability at opposite walls of a chamber with release of these treats. They can reach the feeders directly along a line of site or by an alternate corridor, and the floor 
surface of the shorter, line-of-site route can be altered to presumably be aversive after nerve injury. The preference for taking the short route is a primary outcome measure of the test. The current study examined whether SNL surgery alters the primary outcome measure, whether this test can be performed repeatedly, whether behavioral disruption after SNL in the shuttle maze parallels that of mechanical hypersensitivity in the paw withdrawal test, and whether one of the first-line drugs for neuropathic pain, gabapentin, can restore behavioral disruption and mechanical hypersensitivity similarly in both tests via activation of spinal $\alpha 2$-adrenoceptors.

\section{Materials and methods}

\section{Animals}

Eighty-three male Sprague-Dawley rats (Japan SLC, Hamamatsu, Japan), 5 weeks old on arrival, were housed in pairs under a $12 \mathrm{~h}$ light-dark cycle (light cycle: $7 \mathrm{am}-7 \mathrm{pm}$ ) with free access to food and water, and used for the study after a minimum of 5 days from arrival. All experiments were approved by the Animal Care and Use Committee at Akita University (Akita, Japan).

\section{Shuttle maze apparatus}

Prior to the current study, pilot experiments with 54 rats were performed to develop the shuttle maze apparatus (maze shape and object material) and to determine training and testing conditions. The shuttle maze consisted of two arms (30 cm length) and two routes (short and long) with vinyl chloride floors, which were surrounded by internal and external walls of $20 \mathrm{~cm}$ height (Fig. 1a, b). Treat feeders, made of 50-ml centrifuge tubes, were placed at the end of the direct long-axis corridor and a 20-25 mg piece of chocolate flavor cereal (Coco Choco Crispy, Kellogg's, Tokyo, Japan), as a treat, was dropped from the outside of the wall into the feeder through a pipe. An arch-shaped object $(10 \mathrm{~cm}$ height, $40 \mathrm{~cm}$ length) with a prickly surface (San-step mat, F-131-1, The Yamazaki Corporation, Osaka, Japan) was placed on the short route, necessitating the use of all four limbs to go up and down the object to reach the other side. During the experiment, an acrylic glass was placed over the maze to prevent the animal from escaping, and animal behavior was monitored and recorded using a video camera connected to a computer. The video data were analyzed after completion of each set of experiments. The person who
A
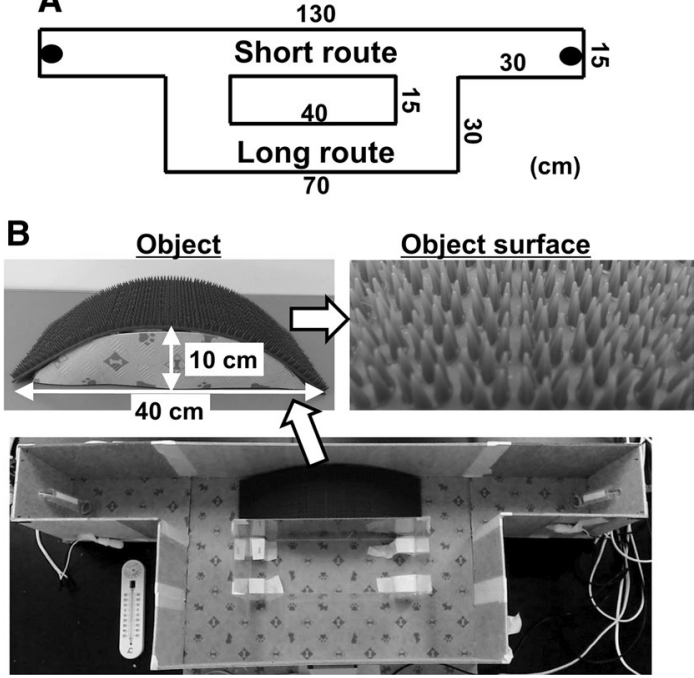

Fig. 1 Shuttle maze apparatus. a Dimension of shuttle maze: the walls of the maze are $20 \mathrm{~cm}$ high and the entire maze is covered during the experiment with an acrylic glass. Black circles at the ends of both arms represent treat feeders. Treats are dropped from outside the wall into the feeder through a pipe. Animals pass through the short route or the long route when moving between the treat feeders. $\mathbf{b}$ Photographs of a prickly surfaced arch object $(10 \mathrm{~cm}$ height $\times 40 \mathrm{~cm}$ length) and its surface, and upper view of the maze

analyzed the video data was blinded to drug treatments but not to surgery and floor conditions.

\section{Shuttle maze training and testing protocols}

Rats had free access to food and water prior to the training and experimental sessions, which were conducted one session per day at 9:00 am-3:00 pm (during the light cycle) under low light $(<30$ lumens) conditions. All rats were trained for 10-12 days prior to the experiments. During the training, the prickly surfaced object was randomly placed on the short or long route and the rat was placed in the arm of the shuttle maze facing toward the feeder with a piece of treat, and allowed to explore the maze for $20 \mathrm{~min}$. When the rat ate the treat, another treat was dropped into the other side so that the rat learned to go back and forth between the treat feeders to obtain treats. To recruit animals with stable task performance during the experiments, only animals ( 76 of 83 rats) that took more than 15 treats at the last training session were used in the current study. On the day of the experiment, treats were alternately placed in the feeders and the number of times that the rat passed the short and long routes, with or without the object, to obtain treats, was counted. The session was stopped at $20 \mathrm{~min}$ or when the rat had shuttled back and forth 20 times (counts). A preference index value for the short route, a percentage of counts that the rat chose the short route over the total counts, was calculated. 


\section{Spinal nerve ligation (SNL) surgery and measurements of withdrawal thresholds}

Unilateral L5 and L6 SNL was performed to induce mechanical hypersensitivity in the right hind paw as described previously [7]. Briefly, after the last shuttle maze training session, 38 animals were anesthetized with $2.0 \%$ isoflurane in oxygen, the right L6 transverse process was removed, and the right L5 and L6 spinal nerves were tightly ligated using 5-0 silk sutures. Eight rats underwent sham surgery with isoflurane anesthesia and surgical exposure without ligation of the right L5 and L6 spinal nerves. Mechanical withdrawal thresholds in the hind paw were measured with a Randall-Selitto analgesimeter (Ugo Basile, Comerio, Italy) as previously described [8]. A cutoff pressure of $250 \mathrm{~g}$ was used to avoid potential tissue injury. All animals were trained for 3 days with this apparatus prior to the experiment. In the current study, all animals showed less than $100 \mathrm{~g}$ postsurgical withdrawal thresholds in the hind paw ipsilateral to SNL. The experimenter who measured withdrawal thresholds was blinded to drug treatments and shuttle maze results but not to surgery.

\section{Drug treatments}

Gabapentin (100 mg/kg in $5 \mathrm{ml}$, Toronto Research Chemicals Inc., Toronto, Canada) was dissolved in water and orally administered by a feeding tube. Idazoxan $(30 \mu \mathrm{g} / \mathrm{rat}$ in $10 \mu \mathrm{l}$, Sigma-Aldrich, St. Louis, MO, USA) was dissolved in saline and injected intrathecally via the L5-L6 intervertebral space under brief $2.5 \%$ sevoflurane anesthesia using a 30-gauge needle as previously described [9]. The doses of gabapentin and idazoxan were determined from our previous studies $[10,11]$.

\section{Experimental design}

Withdrawal threshold values and shuttle maze video data were analyzed after completion of each set of experiments. A diagram showing the numbers of rats used in the different experiments is shown in Fig. 2.

1. Time course changes after SNL in the shuttle maze test with the object on the short route.

The experiments were performed in one cohort (total 12 rats) including six normal and six SNL rats. Shuttle maze tests with the object on the short route were performed once a week before and then once a week until 4 weeks after SNL surgery.

2. Effects of object placement and oral gabapentin in the shuttle maze test.

The experiments were performed in two cohorts with a total of 32 rats. Animals in the first cohort (16 rats)

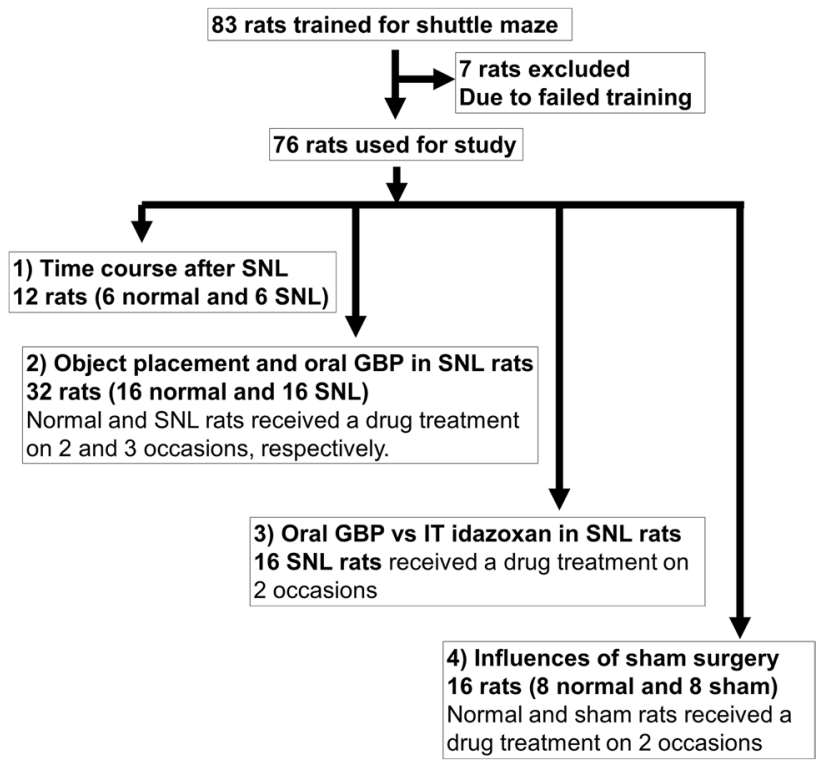

Fig. 2 Animal allocation in the current study. Eighty-three rats were trained in 10-12 sessions (20 min per session per day). The 76 rats that took more than 15 treats at the last training session were used for 4 sets of experiments and 7 rats that took less than 15 treats were excluded. (1) Time course changes after SNL with the object on the short route were examined in one cohort (total 12 rats) including 6 normal and 6 SNL rats. (2) Effects of object placement and oral gabapentin in the shuttle maze test were examined in two cohorts of total 32 rats (16 rats/cohort). In each cohort, animals were randomly assigned to normal and SNL groups (8 rats in each group), and shuttle maze tests were performed starting from 2 weeks after SNL surgery (see "Methods" for detail). (3) Effects of intrathecal idazoxan in SNL animals treated with oral gabapentin in the shuttle maze test were examined in one cohort of total 16 rats. (4) Influences of sham surgery on preference index and gabapentin's effect in the shuttle maze test were examined in one cohort (total 16 rats) including 8 normal and 8 sham rats

were randomly assigned to normal and SNL groups (eight rats in each group), and shuttle maze tests were performed 2 weeks after SNL surgery for 3 days (day 1: no object, day 2: object on short route, day 3: object on long route). Then, normal animals were randomly assigned to receive an oral administration of one of two treatments (vehicle and gabapentin $100 \mathrm{mg} / \mathrm{kg}$ ) $2 \mathrm{~h}$ prior to shuttle maze testing with or without the object on the short route on two occasions separated by 4 days. SNL animals were randomly assigned to receive an oral administration of one of four treatments (vehicle, gabapentin 10,30 , and $100 \mathrm{mg} / \mathrm{kg}$ ) $2 \mathrm{~h}$ prior to shuttle maze testing with or without the object on the short route on three occasions separated by 4 days. The same experiments were performed in the second cohort (16 rats).

3. Effects of intrathecal idazoxan in SNL animals treated with oral gabapentin.

The experiments were performed in one cohort (16 SNL rats). Animals randomly received oral vehicle or 
gabapentin $(100 \mathrm{mg} / \mathrm{kg}) 2 \mathrm{~h}$ prior to and intrathecal saline or idazoxan $(30 \mu \mathrm{g} / \mathrm{rat}) 30 \mathrm{~min}$ prior to shuttle maze tests with the object on the short route on two occasions separated by 4 days.

4. Influences of sham surgery in shuttle maze test.

The experiments were performed in one cohort (total 16 rats) including eight normal and eight sham rats, and shuttle maze tests were performed 2 weeks after sham surgery. Animals were randomly assigned to receive an oral administration of one of two treatments (vehicle and gabapentin $100 \mathrm{mg} / \mathrm{kg}$ ) $2 \mathrm{~h}$ prior to shuttle maze testing with the object on the short route on two occasions separated by 4 days.

\section{Statistical analysis}

We determined the sample size for the preceding experiments based on our experience in the pilot experiments and a priori power analysis was not performed. Unless otherwise stated, data are presented as mean \pm SD. Shuttle maze time course data were analyzed by two-way repeated measures analysis of variance (ANOVA) using SigmaPlot software (Systat Software Inc, Chicago, IL, USA). Other data were analyzed by one-way or two-way ANOVA followed by Dunnett's post hoc test. $p<0.05$ adjusted by SigmaPlot software was considered significant.

\section{Results}

\section{Time course changes after SNL in the shuttle maze test with the object on the short route}

There were main effects of surgery $\left(F_{1,40}=541.52\right.$; $p<0.01)$, time $\left(F_{4.40}=12.28 ; p<0.01\right)$, and surgery $\times$ time interaction $\left(F_{4,40}=11.47 ; p<0.01\right)$ on withdrawal thresholds in the hind paw ipsilateral to surgery (Fig. 3a). There were main effects of surgery $\left(F_{1,40}=47.40 ; p<0.01\right)$, time $\left(F_{4,40}=4.99 ; p<0.01\right)$, and surgery $\times$ time interaction $\left(F_{4,40}=3.03 ; p=0.03\right)$ on the preference index (Fig. 3b). Post hoc testing revealed that SNL resulted in mechanical hypersensitivity in the hind paw ipsilateral to injury and reduced the preference index for the short route with the object from 1 to 4 weeks after surgery. All normal and SNL rats completed 20 counts (treat deliveries) in all time points of this experiment. On the basis of these results, we selected a sample size of eight rats to examine effects of test drugs on the preference index and time points of 2-3 weeks after SNL surgery for subsequent experiments.

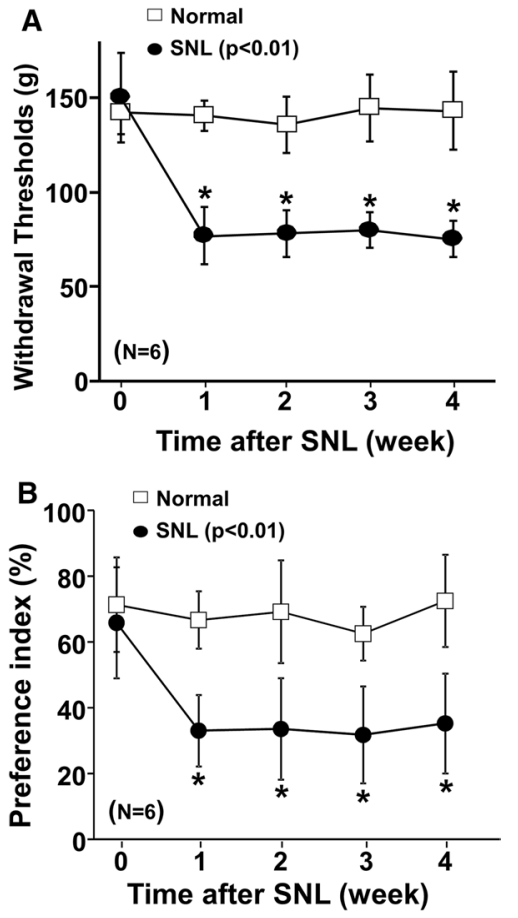

Fig. 3 Time course changes in withdrawal thresholds and preference index with the object on the short route after SNL. a Withdrawal thresholds in the hind paw ipsilateral to SNL were measured in normal and SNL rats 0-4 weeks after surgery. b Preference index values are presented as the percentage of counts that animals chose the short route over the total counts. Groups differ by two-way ANOVA for repeated measures. ${ }^{*} p<0.01$ vs normal

\section{Effects of object placement and oral gabapentin in the shuttle maze test}

\section{Effects of object placement}

Neither SNL surgery nor object placement affected the total treat counts (Fig. 4a). In contrast, there were main effects of surgery $\left(F_{190}=6.65 ; p=0.01\right)$, object placement $\left(F_{2,90}=64.54 ; p<0.01\right)$, and surgery $\times$ object placement interaction $\left(F_{2,90}=24.16 ; p<0.01\right)$ on the preference index (Fig. 4b). Post hoc testing revealed that SNL significantly reduced the preference index when the object was placed on the short route compared with the normal $(p<0.01)$ and that object placement on the short route significantly reduced the preference index compared with no object condition in SNL rats $(p<0.01)$.

There was a significant correlation between withdrawal thresholds in the hind paw ipsilateral to surgery and preference index for the short route with the object in SNL rats $(p=0.01)$ but not in normal rats $(p=0.62)$ (Fig. $4 \mathrm{c})$.

1. Effects of gabapentin in the presence of the object on the short route. 


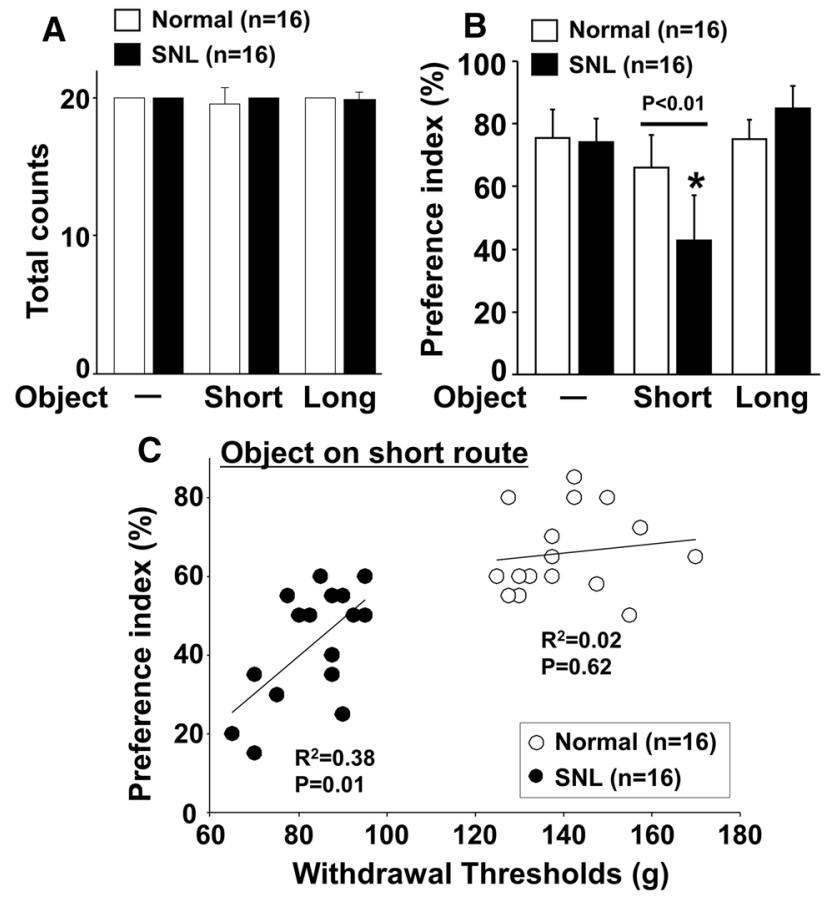

Fig. 4 Effects of object placement in shuttle maze test and relationship between hypersensitivity and preference index after SNL surgery. Shuttle maze measurements were performed in normal and SNL rats 2 weeks after surgery for 3 consecutive days (day 1: no object, day 2: object on short route, day 3: object on long route). a Total counts and $\mathbf{b}$ preference index with or without the object on the short or long route. Preference index values are presented as the percentage of counts that animals chose the short route over the total counts. ${ }^{*} p<0.01$ vs no object. $\mathbf{c}$ The relationship between withdrawal thresholds in the hind paw ipsilateral to nerve injury and preference index values with the object on the short route in normal and SNL rats 2 weeks after surgery

In normal rats, the preference index with the object on the short route $(p=0.63)$ and the changes in withdrawal threshold values $(p=0.49)$ did not differ between vehicle and gabapentin $(100 \mathrm{mg} / \mathrm{kg})$ treated groups (Fig. 5a, b). In SNL rats, there was a main effect of gabapentin on the preference index with the object on the short route $\left(F_{3.28}=8.40 ; p<0.01\right)$ (Fig. 5 c, d). Post hoc testing revealed that gabapentin significantly increased the preference index on the short route with the object at the doses of $30 \mathrm{mg} / \mathrm{kg}(p=0.01)$ and $100 \mathrm{mg} / \mathrm{kg}(p<0.01)$ compared to vehicle in SNL rats. Similarly, there was a main effect of gabapentin treatment on the changes in withdrawal thresholds in SNL rats $(p<0.01)$. Post hoc testing revealed that gabapentin at a dose of $100 \mathrm{mg} / \mathrm{kg}$ significantly increased withdrawal thresholds compared to vehicle in SNL rats $(p<0.01)$. In all treatment groups, all rats completed 20 counts during the shuttle maze testing with the object on the short route.

2. Effects of gabapentin in the absence of the object.
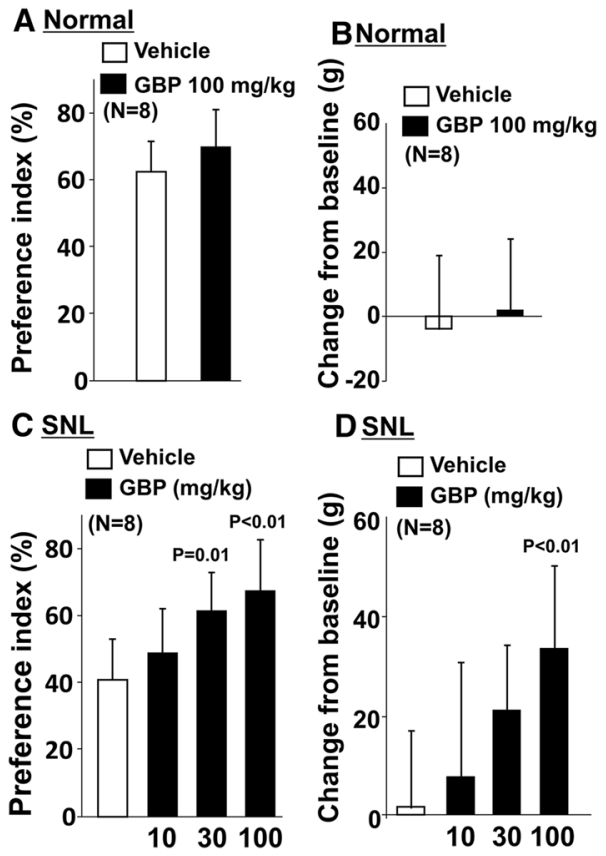

Fig. 5 Effects of gabapentin on preference index with the object on the short route and hypersensitivity after SNL surgery. a, b Normal and c, d SNL rats 2-3 weeks after surgery received an oral administration of vehicle or gabapentin (GBP, 10, 30, or $100 \mathrm{mg} / \mathrm{kg}$ ) $2 \mathrm{~h}$ prior to shuttle maze measurement with the object on the short route. Preference index values are presented as the percentage of counts that animals chose the short route over the total counts $(\mathbf{a}, \mathbf{c})$. Withdrawal thresholds in the hind paw ipsilateral to nerve injury were measured prior to the drug administration (baseline) and just after the shuttle maze measurement in normal and SNL rats $(\mathbf{b}, \mathbf{d})$. Data are presented as changes from the baseline

In normal rats, the preference index without the object $(p=0.57)$ and the changes in withdrawal threshold values $(p=0.82)$ did not differ between vehicle and gabapentin $(100 \mathrm{mg} / \mathrm{kg})$ treated groups (Fig. 6a, b). In SNL rats, gabapentin significantly increased withdrawal thresholds $(p<0.01)$ but failed to affect the preference index without the object $(p=0.48)$ compared to vehicle (Fig. 6c, d). In all treatment groups, all rats completed 20 counts during shuttle maze testing without the object.

\section{Effects of intrathecal idazoxan in SNL animals treated with oral gabapentin}

We and others previously demonstrated in rodents after peripheral nerve injury that gabapentin activates descending noradrenergic inhibition to reduce hypersensitivity via stimulation of spinal $\alpha 2$-adrenoceptors, using paw withdrawal threshold measurements $[11,12]$. We therefore examined whether the efficacy of gabapentin on the preference index relies on activation of spinal $\alpha 2$-adrenoceptors by testing the effects of orally administered gabapentin $(100 \mathrm{mg} / \mathrm{kg})$ with an intrathecal injection 


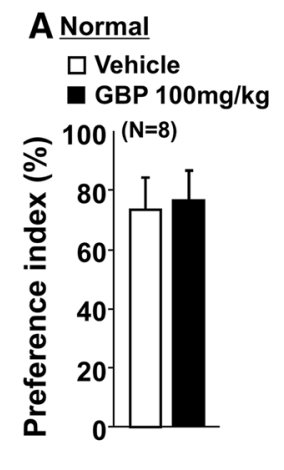

\section{B Normal \\ $\square$ Vehicle \\ GBP $100 \mathrm{mg} / \mathrm{kg}$}
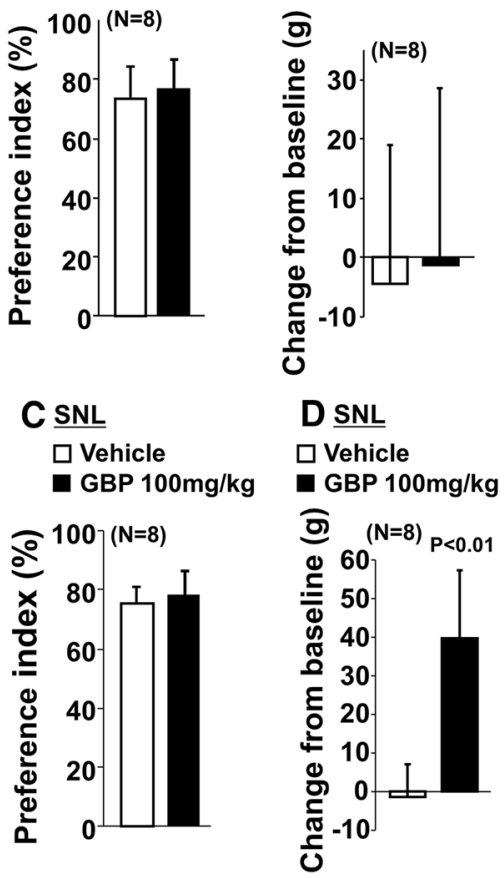

Fig. 6 Effects of gabapentin on preference index without the object and hypersensitivity after SNL surgery. a, b Normal and c, d SNL rats $2-3$ weeks after surgery received an oral administration of vehicle or gabapentin (GBP, $100 \mathrm{mg} / \mathrm{kg}$ ) $2 \mathrm{~h}$ prior to shuttle maze measurement without the object. Preference index values are presented as the percentage of counts that animals chose the short route over the total counts $(\mathbf{a}, \mathbf{c})$. Withdrawal thresholds in the hind paw ipsilateral to nerve injury were measured prior to the drug administration (baseline) and just after the shuttle maze measurement in normal and SNL rats $(\mathbf{b}, \mathbf{d})$. Data are presented as changes from the baseline

of the $\alpha 2$-adrenoceptor blocker idazoxan (30 $\mu \mathrm{g} / \mathrm{rat})$ in SNL rats. Total treat counts did not differ among the treatment groups (Fig. 7a). In the preference index with the object on the short route (Fig. $7 \mathrm{~b}$ ), there were main effects of intrathecal idazoxan $\left(F_{1,28}=12.44 ; p<0.01\right)$ and oral gabapentin $\left(F_{1,28}=17.70 ; p<0.01\right)$, but no gabapentin $\times$ idazoxan interaction $\left(F_{1,28}=3.66 ; p=0.07\right)$. In the changes in withdrawal thresholds (Fig. 7c), there were main effects of intrathecal idazoxan $\left(F_{1,28}=15.99\right.$; $p<0.01)$, oral gabapentin $\left(F_{1,28}=27.60 ; p<0.01\right)$, and gabapentin $\times$ idazoxan interaction $\left(F_{1,28}=9.84 ; p<0.01\right)$. Post hoc testing revealed that oral gabapentin significantly increased withdrawal thresholds compared to oral vehicle in intrathecal saline treated groups $(p<0.01)$ and that intrathecal idazoxan significantly decreased gabapentin's effect on withdrawal thresholds compared to intrathecal saline $(p<0.01)$.
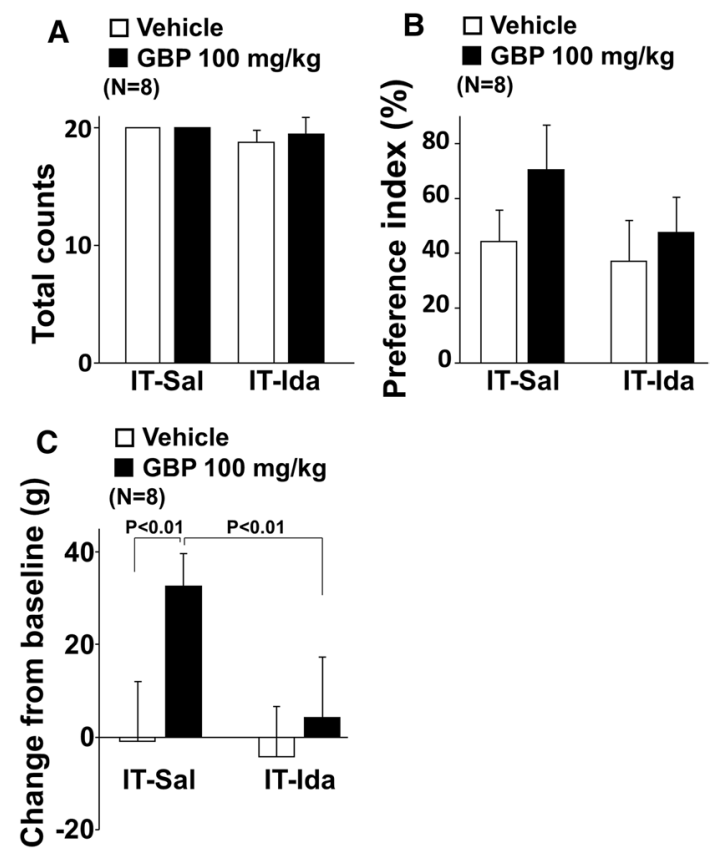

Fig. 7 Role of spinal noradrenergic signaling in increased preference index and antihypersensitivity effect from oral gabapentin in SNL rats. Oral vehicle or gabapentin (GBP, $100 \mathrm{mg} / \mathrm{kg}$ ) were administered $2 \mathrm{~h}$ prior to and intrathecal saline (IT-Sal) or idazoxan (IT-Ida, $30 \mu \mathrm{g} / \mathrm{rat}$ ) were administered $30 \mathrm{~min}$ prior to the shuttle maze measurement with the object on the short route in SNL rats 2-3 weeks after surgery. a Total counts during the shuttle maze measurement. b Preference index values are presented as the percentage of counts that animals chose the short route over the total counts. $\mathbf{c}$ Withdrawal thresholds in the hind paw ipsilateral to nerve injury were measured prior to the drug administration (baseline) and just after the shuttle maze measurement in SNL rats. Data are presented as changes from the baseline

\section{Influences of sham surgery in shuttle maze test}

Since the SNL procedure is invasive and likely to induce tissue inflammation, surgical intervention rather than nerve injury might have some effects on shuttle maze behavior and affect the results of gabapentin experiments. We therefore examined whether sham surgery without nerve injury affects the preference index and whether gabapentin alters that of sham-operated rats, by testing the effects of orally administered vehicle or gabapentin $(100 \mathrm{mg} / \mathrm{kg})$ in normal and sham rats. In all treatment groups, all rats completed 20 counts during the shuttle maze testing with the object on the short route. Withdrawal thresholds in the hind paw ipsilateral to sham surgery did not differ between normal $(144 \pm 13 \mathrm{~g}, n=8)$ and sham $(140 \pm 15 \mathrm{~g}, n=8)$ rats. In the preference index with the object on the short route (Fig. 8a), there was no main effect of sham surgery $\left(F_{1,28}=0.17 ; p=0.68\right)$, gabapentin treatment $\left(F_{1,28}=1.91\right.$; $p=0.18$ ), or sham surgery $\times$ gabapentin treatment interaction $\left(F_{1,28}=0.31 ; p=0.59\right)$. Similarly, in the changes in 


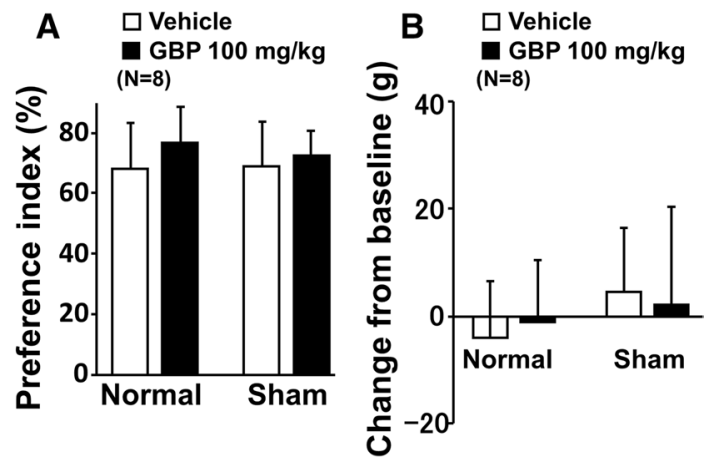

Fig. 8 Effects of gabapentin on preference index with the object on the short route and withdrawal thresholds after sham surgery. a Normal and sham rats 2 weeks after surgery received an oral administration of vehicle or gabapentin (GBP, $100 \mathrm{mg} / \mathrm{kg}$ ) $2 \mathrm{~h}$ prior to shuttle maze measurement with the object on the short route. Preference index values are presented as the percentage of counts that animals chose the short route over the total counts. b Withdrawal thresholds in the hind paw ipsilateral to sham surgery were measured prior to the drug administration (baseline) and just after the shuttle maze measurement in normal and sham rats. Data are presented as changes from the baseline

withdrawal thresholds (Fig. 8b), there was no main effect of sham surgery $\left(F_{1,28}=1.60 ; p=0.22\right)$, gabapentin treatment $\left(F_{1,28}=0.004 ; p=0.95\right)$, or sham surgery $\times$ gabapentin treatment interaction $\left(F_{1,28}=0.36 ; p=0.55\right)$. These results indicate no influence of sham surgery in the current shuttle maze study.

\section{Discussion}

Pain is a subjective experience which non-human animals cannot self-report and which cannot be unambiguously inferred by reflexive behaviors in response to stimuli. The current study presents a novel method that combines motivated behavior and an environment which can be manipulated to present stimuli which are presumed to be nociceptive only in the presence of injury, and which can be performed in the absence of investigator presented stimulation or external stressors.

In the current study, the training failure rate was $8.4 \%$. Since those rats were free-fed prior to the experiment, they presumably performed the shuttle maze task based on their motivation for the chocolate flavored cereal. Food restriction would be expected to increase the motivation of rats to perform the task and thereby could reduce the number of excluded rats. However, since food restriction alters nociceptive thresholds and increases stress hormone in rats [13, 14], we chose a free-feeding condition in the current study. We did not perform a priori power analysis to determine appropriate sample sizes for the current experiments. Post hoc sample size calculation by SigmaPlot software using the current preference index results in SNL rats 2 weeks after surgery (SD:15\%) shows that nine samples per group are required in order to detect a minimal difference of $20 \%$ in the preference index with an alpha level of 0.05 and power of 0.8 .

A previous study in rats demonstrated that peripheral nerve injury reduced voluntary activity in wheel-running [15]. We therefore expected that SNL surgery might also reduce the activity in the shuttle maze assay. In contrast to our expectation, nerve injury failed to alter task activity of rats up to 4 weeks after surgery in the current study. This discrepancy may be due to differences in strength of motivation and reward (running vs chocolate flavored cereal) and sites of nerve injury (sciatic nerve vs spinal nerve).

Operant measurements of pain using conditioned place aversion, such as conditioned place avoidance [16] and the PEAP [3], have been used to assess aversion to noxious stimuli. Particularly, the PEAP made a breakthrough in the assessment of the unpleasant component of evoked pain in rodents $[3,4]$. However, during the PEAP, animals keep receiving forced stimulations to the paw by the experimenter and may perform the task based on fear or anxiety against pain or unpleasantness. The stress of this paradigm could affect nociceptive thresholds and mask pain behavioral expression [5, 6]. On the other hand, during the shuttle maze task, animals do not receive any forced stimulation and they have the choice of taking either the route with or without a prickly surfaced arch. Importantly, the animals do not have any painful consequences of not performing the task in the shuttle maze, and presumably this assay is less stressful than PEAP.

We chose an arch-shaped object rather than a flat experimental surface so that animals had to bear weight on all four extremities to traverse the object. In the absence of the object, animals showed a similar preference for the short route in normal and SNL conditions, confirming that nerve injury does not alter natural behavior of animals to choose a short distance when moving between the places. When the object was placed on the short route, SNL resulted in a greater reduction of the preference index compared to the normal condition, correlated with mechanical hypersensitivity. That this effect of SNL was reversed by gabapentin argues that the reason for this change in preference reflects an aversion to weight bearing on a prickly surfaced floor with the hypersensitive paw ipsilateral to injury. When the object was placed on the long route, both normal and SNL rats showed around $80 \%$ of the preference index for the short route, presumably indicating a ceiling of the preference index in the current experimental setting.

Gabapentin has been shown to reduce pain by its actions in the spinal cord, where it reduces primary afferent traffic and excitation of spinal nociceptive neurons, via interaction with $\alpha 2 \delta$ subunits of voltage-gated $\mathrm{Ca}^{2+}$ channels 
[17-19]. However, analgesic efficacy of gabapentin may not rely exclusively on its spinal mechanisms, since intrathecal gabapentin shows no clinical efficacy in patients with chronic pain [20], despite the known efficacy of oral gabapentin in this patient population. We and others have proposed the role of descending inhibition for gabapentin's efficacy by demonstrating in rodents after peripheral nerve injury that gabapentin activates noradrenergic neurons in the locus coeruleus to induce spinal noradrenaline release, and that spinal $\alpha 2$-adrenoceptor antagonism can nearly abolish the antihypersensitivity effect of gabapentin in the paw withdrawal measurements $[12,21]$, consistent with the current result. In contrast, the intrathecal $\alpha 2$-adrenoceptor antagonist idazoxan failed to reverse the gabapentin's effect on the preference index in SNL rats $(p=0.07)$. Unlike the paw withdrawal response, which mainly relies on spinal reflexes, the shuttle maze behavior involves cognitive and motivational functions in the brain, where gabapentin has been shown to act for pain relief [22]. Therefore, not only descending inhibitory pathways but also multiple sites at the spinal and supraspinal levels may contribute to gabapentin's efficacy in the shuttle maze test. In addition, the current results would raise a caution against the overestimation of paw withdrawal measurements for studying therapeutic mechanisms of analgesics.

In summary, the current study demonstrated that SNL injury alters behavior in the shuttle maze test without forced stimulation and that the shuttle maze test shows comparable results to reflexive mechanical hypersensitivity after nerve injury in magnitude and response to gabapentin.

Acknowledgements This work was supported by Grant-in-Aid for Scientific Research (16K08985 and 19K09343, Tokyo, Japan) to Ken-ichiro Hayashida, and by the National Institutes of Health (P01 GM113852, Bethesda, MD, USA) to Thomas Martin.

Author contributions Ken-ichiro Hayashida developed the maze apparatus, designed and conducted the study, analyzed and interpreted the data, and wrote the manuscript. Thomas Martin developed the maze apparatus, designed and conducted the study, interpreted the data, and edited the manuscript. James Eisenach and Masahito Kawatani designed the study, interpreted the data, and edited the manuscript.

\section{Compliance with ethical standards}

Conflict of interest James Eisenach has served as a consultant to Adynxx (San Francisco, CA, USA) and TEVA Pharmaceutical Industries (Petah Tikvah, Israel) regarding preclinical and clinical analgesic development.

\section{References}

1. Gregory NS, Harris AL, Robinson CR, Dougherty PM, Fuchs PN, Sluka KA (2013) An overview of animal models of pain: disease models and outcome measures. J Pain 14:1255-1269. https://doi. org/10.1016/j.jpain.2013.06.008

2. Chesler EJ, Wilson SG, Lariviere WR, Rodriguez-Zas SL, Mogil JS (2002) Influences of laboratory environment on behavior. Nat Neurosci 5:1101-1102. https://doi.org/10.1038/nn1102-1101

3. LaBuda CJ, Fuchs PN (2000) A behavioral test paradigm to measure the aversive quality of inflammatory and neuropathic pain in rats. Exp Neurol 163:490-494. https://doi.org/10.1006/ exnr.2000.7395

4. Pratt D, Fuchs PN, Sluka KA (2013) Assessment of avoidance behaviors in mouse models of muscle pain. Neuroscience 248:54 60. https://doi.org/10.1016/j.neuroscience.2013.05.058

5. Amit Z, Galina ZH (1986) Stress-induced analgesia: adaptive pain suppression. Physiol Rev 66:1091-1120

6. Keogh E, Cochrane M (2002) Anxiety sensitivity, cognitive biases, and the experience of pain. J Pain 3:320-329

7. Kim SH, Chung JM (1992) An experimental model for peripheral neuropathy produced by segmental spinal nerve ligation in the rat. Pain 50:355-363

8. Randall LO, Selitto JJ (1957) A method for measurement of analgesic activity on inflamed tissue. Arch Int Pharmacodyn Ther 111:409-419

9. Kimura M, Eisenach JC, Hayashida K (2016) Gabapentin loses efficacy over time after nerve injury in rats: role of glutamate transporter-1 in the locus coeruleus. Pain 157:2024-2032. https ://doi.org/10.1097/j.pain.0000000000000608

10. Hayashida K, Eisenach JC (2008) Multiplicative interactions to enhance gabapentin to treat neuropathic pain. Eur J Pharmacol 598:21-26. https://doi.org/10.1016/j.ejphar.2008.09.004

11. Hayashida K, Parker R, Eisenach JC (2007) Oral gabapentin activates spinal cholinergic circuits to reduce hypersensitivity after peripheral nerve injury and interacts synergistically with oral donepezil. Anesthesiology 106:1213-1219. https://doi. org/10.1097/01.anes.0000267605.40258.98

12. Tanabe M, Takasu K, Takeuchi Y, Ono H (2008) Pain relief by gabapentin and pregabalin via supraspinal mechanisms after peripheral nerve injury. J Neurosci Res 86:3258-3264. https:// doi.org/10.1002/jnr.21786

13. Hamm RJ, Lyeth BG (1984) Nociceptive thresholds following food restriction and return to free-feeding. Physiol Behav 33:499-501

14. Jurcovicova J, Stancikova M, Svik K, Ondrejickova Krsova D, Seres J, Rokyta R (2001) Stress of chronic food restriction attenuates the development of adjuvant arthritis in male Long Evans rats. Clin Exp Rheumatol 19:371-376

15. Whitehead RA, Lam NL, Sun MS, Sanchez J, Noor S, Vanderwall AG, Petersen TR, Martin HB, Milligan ED (2017) Chronic sciatic neuropathy in rat reduces voluntary wheel-running activity with concurrent chronic mechanical allodynia. Anesth Analg 124:346-355. https://doi.org/10.1213/ANE.0000000000001662

16. Johansen JP, Fields HL, Manning BH (2001) The affective component of pain in rodents: direct evidence for a contribution of the anterior cingulate cortex. Proc Natl Acad Sci USA 98:8077-8082. https://doi.org/10.1073/pnas.141218998

17. Hendrich J, Van Minh AT, Heblich F, Nieto-Rostro M, Watschinger K, Striessnig J, Wratten J, Davies A, Dolphin AC (2008) Pharmacological disruption of calcium channel trafficking by the alpha2delta ligand gabapentin. Proc Natl Acad Sci USA 105:3628-3633. https://doi.org/10.1073/pnas.0708930105

18. Li CY, Zhang XL, Matthews EA, Li KW, Kurwa A, Boroujerdi A, Gross J, Gold MS, Dickenson AH, Feng G, Luo ZD (2006) Calcium channel alpha2delta1 subunit mediates spinal hyperexcitability in pain modulation. Pain 125:20-34. https://doi.org/10.1016/j. pain.2006.04.022

19. Luo ZD, Calcutt NA, Higuera ES, Valder CR, Song YH, Svensson CI, Myers RR (2002) Injury type-specific calcium channel alpha 
2 delta-1 subunit up-regulation in rat neuropathic pain models correlates with antiallodynic effects of gabapentin. J Pharmacol Exp Ther 303:1199-1205. https://doi.org/10.1124/jpet.102.04157 4

20. Rauck R, Coffey RJ, Schultz DM, Wallace MS, Webster LR, McCarville SE, Grigsby EJ, Page LM (2013) Intrathecal gabapentin to treat chronic intractable noncancer pain. Anesthesiology 119:675-686. https://doi.org/10.1097/ALN.0b013e3182a10fbf

21. Hayashida K, Obata H, Nakajima K, Eisenach JC (2008) Gabapentin acts within the locus coeruleus to alleviate neuropathic pain. Anesthesiology 109:1077-1084. https://doi.org/10.1097/ ALN.0b013e31818dac9c
22. Bannister K, Qu C, Navratilova E, Oyarzo J, Xie JY, King T, Dickenson AH, Porreca F (2017) Multiple sites and actions of gabapentin-induced relief of ongoing experimental neuropathic pain. Pain 158:2386-2395. https://doi.org/10.1097/j.pain.00000 00000001040

Publisher's Note Springer Nature remains neutral with regard to jurisdictional claims in published maps and institutional affiliations. 